УДК 541.123.3

\title{
ЗАВИСИМОСТЬ ТЕРМОДИНАМИЧЕСКИХ СВОЙСТВ Н-АЛКИЛКЕТОНОВ ОТ ТЕМПЕРАТУРЫ ВСПЫШКИ
}

\author{
(C) 2018 Ю. К. Сунцов ${ }^{1}$ Ю. Н. Сорокина ${ }^{2}$, Е. В. Шумилин ${ }^{1}$ \\ ${ }^{1}$ Воронежский государственный педагогический университет, ул. Ленина, 86, 394043 Воронеж, Россия \\ ${ }^{2}$ Воронежский институт-филиал Ивановской пожарно-спасательной академии ГПС МЧС России, \\ ул. Краснознамённая, 231, 394052 Воронеж, Россия \\ e-mail:jsyntsov@mail.ru,sorokina-jn@mail.ru
}

Поступила в редакцию 24.10.2018

\begin{abstract}
Аннотация. Эбулиометрическим методом при различных давлениях измерены температуры кипения пяти веществ гомологического ряда н-алкилкетонов. На основе опытных данных с использованием стандарта идеального газа рассчитаны значения внутренней энергии, энтропии и энергии Гельмгольца для веществ гомологического ряда н-алкилкетонов. Установлено, что значения термодинамических функций веществ аддитивно зависят от молярной массы н-алкилкетона в гомологическом ряду. Анализом литературных данных обнаружена также аддитивная зависимость температур вспышки веществ от молярной массы н-алкилкетонов. Анализом установлено, что значения энергии Гельмгольца линейно зависят от температуры вспышки н-алкилкетона в гомологическом ряду. Приведены необходимые для расчётов термодинамических свойств веществ уравнения.
\end{abstract}

Ключевые слова: н-алкилкетоны, равновесие жидкость-пар, энергия Гельмгольца, внутренняя энергия, энтропия, температура вспышки, уравнение состояния.

DOI: DOI: https://doi.org/10.17308/kcmf.2018.20/641

\section{ВВЕДЕНИЕ}

Термодинамические свойства веществ являются основой теоретически достижимых технологических показателей и движущих сил, определяющих направление и скорость протекания технологических процессов. Для технических расчётов процессов и аппаратов производства н-алкилкетонов необходимы данные о термодинамических свойствах и показателях пожаровзрывоопасности веществ. К числу важнейших показателей пожаровзрывоопасности относятся температуры вспышки жидкостей, которые можно рассчитать на основе исследований фазовых равновесий жидкость-пар [1]. Известно, что исследование термодинамических свойств веществ и фазовых равновесий связано с большими экспериментальными трудностями [2]. Поэтому необходимость разработки метода прогнозирования термодинамических свойств и показателей пожаровзрывоопасности веществ, исходя из минимального количества экспериментальных данных, является актуальной задачей. Решение этой задачи связано с установлением взаимосвязи между термодинамическими свойствами и молекулярными характеристиками веществ в гомологических рядах.

Ранее была установлена взаимосвязь температуры вспышки и энергии Гельмгольца с молярной массой веществ для гомологических рядов н-алкилэтаноатов, н-алкилпропаноатов, н-алкилбутаноатов, н-спиртов, н-алкиламинов, н-алкилбензолов [3-6]. В данной работе предпринята попытка установления подобных аналитических зависимостей для веществ гомологического ряда н-алкилкетонов.

\section{ЭКСПЕРИМЕНТАЛЬНАЯ ЧАСТЬ}

В качестве объектов исследования выбраны вещества: метилэтилкетон, диэтилкетон, пропилэтилкетон, бутилэтилкетон, гексилэтилкетон. Константы очищенных веществ удовлетворительно совпадали с литературными данными [7]. Температуры кипения растворов $(T)$ измерялись эбулиометрическим методом при пониженных давлениях $(P)$ платиновым термометром сопротивления с точностью $\pm 0.05 \mathrm{~K}$. Давление насыщенного пара растворов $(P)$ измерялось ртутным манометром с использо- 
ванием катетометра В-630 с точностью \pm 6.66 Па. Постоянство давления в эбулиометрах поддерживалось изодромным регулятором с отрицательной обратной связью с точностью \pm 6.66 Па. Плотность чистых веществ и их растворов измерялась с помощью пикнометров Оствальда объёмом $50 \mathrm{~cm}^{3}$ при различных температурах. Детальное описание экспериментальных установок и методик эксперимента приведено в [8].

Для расчётов вкладов межмолекулярного взаимодействия в термодинамические функции использовали стандарт идеального газа, взятого при температуре $(T)$ и объёме $(V)$ реальной жидкости. В случае перехода моля вещества из состояния идеального газа в жидкое состояние (с межмолекулярным взаимодействием) получили [8]:

$$
\begin{gathered}
\hat{F}=R T \ln \frac{R T}{P V}-R T+P V ; \\
\hat{U}=-T^{2}\left(\frac{\partial(\hat{F} / T)}{\partial T}\right)_{V, X}=H-R T+P V ; \\
\hat{S}=-\left(\frac{\hat{F}}{\partial T}\right)_{V, X}=H-R T \ln \frac{R T}{P V},
\end{gathered}
$$

где $\hat{F}$ - энергия Гельмгольца; $R$ - универсальная газовая постоянная; $T$ - температура, $\mathrm{K} ; P_{2} V$ - давление пара и мольный объем жидкости; $\hat{U}$ - внутренняя энергия; $H$ - мольная энтальпия испарения жидкости; $\hat{S}$ - энтропия. Значения энтальпии испарения $H$ в уравнениях (2) и (3) рассчитывались по уравнению Клаузиуса-Клайперона с использованием зависимости давления насыщенного пара от температуры веществ [2].

\section{РЕЗУЛЬТАТЫ И ИХ ОБСУЖДЕНИЕ}

Температура вспышки, принятая за основу классификации жидкостей по степени их пожаровзрывоопасности, является одним из важнейших показателей, определяющих категоричность процесса, используемого оборудования, зданий и сооружений. Анализом литературных данных [9] установлено, что температура вспышки н-алкилкетонов аддитивно возрастает с увеличением молярной массы (числа групп - $\mathrm{CH}_{2}-$ в молекуле) вещества в гомологическом ряду:

$$
T_{\text {всп }}=1.051 M+190.5, R^{2}=0.9994
$$

где $T_{\text {всп }}$ - температура вспышки, К; $M$ - молярная масса н-алкилкетона.

На основе экспериментальных и литературных данных [7] по уравнениям (1) - (3) при температуpax вспышки рассчитаны значения $F, \hat{U}$ и $\hat{S}$ жид- ких н-алкилкетонов в гомологическом ряду. Оказалось, что энергия Гельмгольца $\hat{F}$, внутренняя энергия $\hat{U}$ и «связанная энергия» - $T \hat{S}$, рассчитанные при температуре вспышки н-алкилкетонов, также аддитивно возрастают с увеличением молярной массы вещества (числа групп - $\mathrm{CH}_{2}-$ в молекуле) в гомологическом ряду н-алкилкетонов:

$$
\begin{gathered}
\hat{F}=90.612 M+11911, R^{2}=0.9935 \\
\hat{U}=327.0 M+7480, R^{2}=0.9875 \\
T \hat{S}=236.4 M-4431, R^{2}=0.9822
\end{gathered}
$$

Аддитивную зависимость значений термодинамических функций от молярной массы вещества в гомологическом ряду можно объяснить подобием структурообразования жидких н-алкилкетонов [10]. Поскольку энергия Гельмгольца $\hat{F}$ наиболее точно рассчитывается по экспериментальным данным, представлялось целесообразным установить связь этой функции с мольным объемом $(V)$ и давлением $(P)$ насыщенного пара веществ гомологического ряда. Корреляционным анализом установлено, что величины $\ln P, V$ линейно зависят от значений $\hat{F}$ веществ в гомологическом ряду и могут быть описаны уравнениями вида:

$$
\begin{gathered}
\hat{F}=-6873 \ln P+71299, R^{2}=0.9902 ; \\
\hat{F}=-67.196 V+12683, R^{2}=0.9942 .
\end{gathered}
$$

Возрастание величины $\hat{F}$, характеризующей межмолекулярные взаимодействия в жидкости, затрудняет переход молекул веществ в паровую фазу и уменьшает по экспоненциальной зависимости давление насыщенного пара жидкости. Дискретное возрастание мольного объёма н-алкилкетона в гомологическом ряду связано с дискретным возрастанием значений энергии Гельмгольца, что может быть объяснено подобием структурообразования жидких н-алкилкетонов.

С учётом установленных закономерностей изменения значений термодинамических функций и температуры вспышки от молярной массы н-алкил кетонов (уравнения (4) - (9)), представлялось целесообразным установить взаимосвязь энергии Гельмгольца, внутренней энергии и связанной энергии с температурой вспышки вещества в гомологическом ряду. На рис. 1 показана зависимость энергии Гельмгольца от температуры вспышки в гомологическом ряду исследуемых веществ.

Корреляционным анализом с использованием программы TableCurve 3D v4.0 установлено, что энергия Гельмгольца $\hat{F}$, внутренняя энергия $\hat{U}$ и связанная энергия $T \hat{S}$ линейно зависят от темпе- 


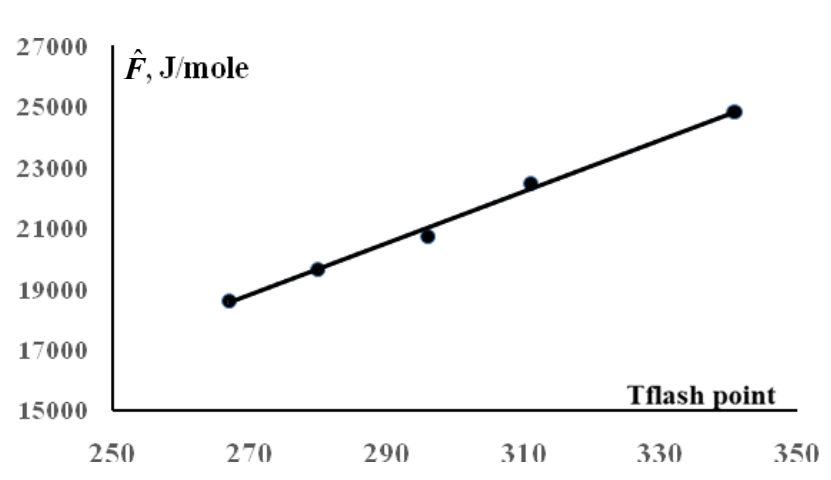

Рис. 1. Зависимость энергии Гельмгольца от температуры вспышки в гомологическом ряду н-алкилкетонов [Fig. 1. Dependence of the Helmholtz energy on the flash point in the homologous series of n-alkylketones]

ратуры вспышки веществ в гомологическом ряду н-алкилкетонов в соответствии с уравнениями:

$$
\begin{gathered}
\hat{F}=85.14 T_{\text {всп }}-4208, R^{2}=0.9954 ; \\
\hat{U}=307.6 T_{\text {всп }}-50803, R^{2}=0.9918 ; \\
T \hat{S}=222.49 T_{\text {всп }}-46596, R^{2}=0.9874 .
\end{gathered}
$$

Уравнения (10-12) с необходимой для технических целей точностью позволяют рассчитывать термодинамические свойства по температурам вспышки н-алкилкетонов и наоборот, используя свойства двух веществ гомологического ряда. Точность вычислений зависит только от точности экспериментальных данных для этих веществ. Ранее подобные закономерности были выявлены для веществ гомологических рядов н-алкилэтаноатов, нспиртов, кетонов, и н-алкиламинов, н-алкилбензолов [3-6]. По мнению авторов, установленные закономерности могут быть применены к веществам других гомологических рядов.

\section{ЗАКЛЮЧЕНИЕ}

1. Установлено, что температуры вспышки налкилкетонов линейно возрастают с увеличением молярной массы (числа групп $-\mathrm{CH}_{2}-$ в молекуле) вещества в гомологическом ряду (уравнение (4)).

2. Рассчитанные при температуре вспышки значения энергии Гельмгольца $\hat{F}$, внутренней энергии $\hat{U}$ и связанной энергии $T \hat{S}$ н-алкилкетонов линейно зависят от молярной массы веществ (числа групп $-\mathrm{CH}_{2}-$ в молекуле) в гомологическом ряду (уравнения (5)-(7)).

3. Установлена взаимосвязь (получены уравнения) значений энергии Гельмгольца, внутренней энергии и связанной энергии с температурой вспышки н-алкилкетонов в гомологическом ряду (уравнения (10)-(12)).

4. Предложена методика, позволяющая прогнозировать термодинамические свойства и температуры вспышки н-алкилкетонов, используя свойства двух веществ гомологического ряда.

6. Установленные соотношения позволяют на термодинамической основе совершенствовать систему государственных отраслевых стандартов, относящихся к номенклатуре показателей пожаровзрывоопасности веществ и материалов и методам их определения.

\section{СПИСОК ЛИТЕРАТУРЫ}

1. Алексеев С. Г., Смирнов В. В., Барбин Н. М. // Пожаровзрывобезопасность, 2012, т. 21, № 10 , c. $21-35$.

2. Морачевский А. Г., Смирнова Н. А., Пиотровская Е. М. и др. Термодинамика равновесия жидкость-nар. Ленинград, Химия, 1989, 344 с.

3. Сунцов Ю. К., Сорокина Ю. Н., Чуйков А. М., Горюнов В. А. // Пожаровзрывобезопасность, 2016, т. 25, № 3, с. 27-33. DOI: https://doi.org/10.18322/ PVB.2016.25.03.27-33

4. Сунцов Ю. К., Сорокина Ю. Н., Чуйков А. М. // Пожаровзрывобезопасность, 2017, т. 26, № 4, c. 21-28. DOI: https://doi.org/10.18322/ PVB.2017.26.04.21-28

5. Чуйков А. М., Сунцов Ю. К., Сорокина Ю. Н., Лукьяненко В. И., Шуткин А. Н. // Вестник Воронежского государственного технического универcumema, 2017, т. 13, № 3, с. 45-49.

6. Сунцов Ю. К., Сорокина Ю. Н. // Конденсированные среды и межфазные границы, 2017, т. 19, № 4, с. 573-576. DOI: https://doi.org/10.17308/ kcmf.2017.19/239

7. Stephenson R. M., Malanowski S. Handbook of the Thermodynamics of Organic Compounds. New York, Elsevier, 1987, 552 p. DOI: https://doi. org/10.1007/978-94-009-3173-2

8. Suntsov Yu. K. // Journal of Chemistry and Chemical Engineering, 2014, vol. 8, № 3, pp. 306-314. DOI: https://doi.org/10.17265/19347375/2014.03.013

9. Корольченко А. Я., Корольченко Д. А. Пожаровзрывоопасность веществ и материалов $и$ средства их тушения. М.: Пожнаука, 2004, ч. I., 713 с.; ч. II., 774 с.

10. Скрышевский А. Ф. Структурнылй анализ жидкостей. М.: Высшая школа, 1971, 256 с. 


\title{
DEPENDENCE OF THE THERMODYNAMIC PROPERTIES OF N-ALKYLKETONES ON THE FLASH POINT
}

\author{
(C) 2018 Yu. K. Suntsov ${ }^{1}$, Yu. N. Sorokina ${ }^{2}$,E. V. Shumilin ${ }^{1}$ \\ ${ }^{1}$ Voronezh State Pedagogical University, 86, Lenin str., 394043 Voronezh, Russia \\ ${ }^{2}$ Voronezh Institute - Branch of the Ivanovo Fire Rescue Academy of State Firefighting Service of EMERCOM of Russia, \\ 231, Krasnoznamennaya str., 394052 Voronezh, Russia \\ e-mail: jsyntsov@mail.ru,sorokina-jn@mail.ru
}

Received 24.10.2018

\begin{abstract}
The number of organic compounds has exceeded 40 million and increases by 300 thousand each year. In order to perform technological calculations of the production process, storage and transportation of materials we need the know the thermodynamic properties and the rate of fire and explosion hazard of the substances used. It is, therefore, important to develop methods of forecasting fire danger indicators and thermodynamic properties of substances, based on a minimal amount of experimental data. To solve this problem it is necessary to determine the relationship between the molecular characteristics and thermodynamic properties of substances in homologous series. The objects of the present study are substances of n-alkylketones homologous series. We have measured the saturation vapour pressure and the density of liquid substances in the series at various temperatures. Using the experimental data and the ideal gas parameters (standard liquid volume and temperature) we calculated the inner energy, the entropy, and the Helmholtz energy. The analysis proved that values of the thermodynamic functions of substances additively depend on the molar weight of n-alkylketones in the homologous series. Existing literature on the problem also demonstrates that similar dependence is observed for the flash point of n-alkylketones. Taking into account the fact that the named properties generally tend to change, we can establish a linear correlation between the flash point values and the Helmholtz energy of the substances in the homologous series. The corresponding equations were obtained. The established regularities and equations based on them, allow us to forecast the properties of homologous series of substances with enough accuracy for practical applications.
\end{abstract}

Keywords: n-alkylketones, liquid-vapor equilibrium, Helmholtz energy, internal energy, entropy; flash point, state equation.

DOI: DOI: https://doi.org/10.17308/kcmf.2018.20/641

\section{REFERENCES}

1. Alekseev S. G., Smirnov V. V., Barbin N. M. Fire and Explosion Safety, 2012, vol. 21, no. 10, pp. 2135. (in Russ.)

2. Morachevskij A. G., Smirnova N. A., Piotrovskaja E. M. Thermodynamics of the Equilibrium of Liquid-Vapor. Leningrad, Chemistry Publ., 1989, 344 p. (in Russ.)

3. Suntcov Ju. K., Sorokina Ju. N., Chujkov A. M., Gorjunov V. A. Fire and Explosion Safety, 2016, vol. 25, no. 3, pp. 27-33. DOI: https://doi.org/10.18322/ PVB.2016.25.03.27-33

4. Suntsov Yu. K., Sorokina Yu. N., Chuykov A. M. Fire and Explosion Safety, 2017, vol. 26, no. 4, pp. 21-28. DOI: https://doi.org/10.18322/PVB.2017.26.04.21-28

5. Chujkov A. M., Suntcov Ju. K., Sorokina Ju. N., Luk'janenko V. I., Shutkin A. N. The Bulletin of Voronezh State Technical University, 2017, vol. 13, no. 3, pp. 45-49. (in Russ.)
6. Suntsov Yu. K., Sorokina Yu. N. Condensed Matter and Interphases, 2017, vol. 19, no. 4, pp. 573-576. DOI: https://doi.org/10.17308/kcmf.2017.19/239

7. Stephenson R. M., Malanowski S. Handbook of the Thermodynamics of Organic Compounds. New York, Elsevier, 1987, 552 p. DOI: https://doi. org/10.1007/978-94-009-3173-2

8. Suntsov Yu. K. Journal of Chemistry and Chemical Engineering, 2014, vol. 8, no. 3, pp. 306-314. DOI: https://doi.org/10.17265/1934- 7375/2014.03.013

9. Korol'chenko, A. Ya., Korol'chenko D. A. Fire and Explosion Hazard of Substances and Materials and Their Means of Extinguishing. Moscow, Pozhnauka Publ., 2004, Pt. I., 713 p.; Pt. II., 774 p. (in Russ.)

10. Skryshevskij A. F. Structural Analysis of Liquids. Moskow, Higher School Publ., 1971, 256 p. (in Russ.) 
Сунцов Юрий Константинович - д. х. н., профессор, заведующий кафедрой безопасности жизнедеятельности, Воронежский государственный педагогический университет, Воронеж, Россия; тел.: +7(950) 7618967, e-mail: syntsov@mail.ru

Сорокина Юлия Николаевна - к. т. н., доцент, доцент кафедры естественно-научных дисциплин, Воронежский институт - филиал Ивановской пожарно-спасательной академии ГПС МЧС России, Воронеж, Россия; тел. +7(906) 5858001; e-mail: sorokina-jn@mail.ru

Шумилин Евгений Витальевич - магистр, Воронежский государственный педагогический университет; Воронеж, Россия; тел.: +7(980) 5584250, e-mail: shum1line@yandex.ru
Yuri K. Suntsov-Dr. Sci. (Chem.), Professor, Head of the Department of Life Safety, Voronezh State Pedagogical University, Voronezh, Russia; tel. +7(950) 7618967, e-mail: syntsov@mail.ru

Yuliya N. Sorokina - Cand. Sci. (Eng.), Associate Professor, Associate Professor of Natural Sciences Department, Voronezh Institute - Branch of the Ivanovo Fire Rescue Academy of State Firefighting Service of EMERCOM of Russia, Voronezh, Russia; tel.: +7(906) 5858001; e-mail: sorokina-jn@mail.ru

Evgeny V. Shumilin - Master Student, Voronezh State Pedagogical University, Voronezh, Russia; tel.: +7(980) 5584250, e-mail: shum1line@yandex.ru 